\title{
The Presence of Pyrrolo Quinoline Quinone (PQQ) in Eukaryotic Tissue Extracts
}

\author{
Flores-Encarnación, M..$^{*}$, Valentín-Aguilar I. ${ }^{1}$, Cabrera-Maldonado C. ${ }^{2}$, Aguilar-Gutiérrez \\ G.R. ${ }^{3}$ \\ ${ }^{1}$ Laboratorio de Microbiología Molecular y Celular. Biomedicina, Facultad de Medicina, Benemérita \\ Universidad Autónoma de Puebla. Puebla. Puebla, México. \\ ${ }^{2}$ Depto. de Microbiología. Facultad de Ciencias Químicas, Benemérita Universidad Autónoma de Puebla. \\ Puebla. Puebla, México. \\ ${ }^{3}$ CICEI. Instituto Nacional de Salud Pública. Cuernavaca, Morelos. México.
}

*Corresponding Author: Flores-Encarnación, M, Laboratorio de Microbiología Molecular y Celular. Biomedicina, Facultad de Medicina, Benemérita Universidad Autónoma de Puebla. Puebla. Puebla, México.

Abstract: $P Q Q$ is a novel quinone, which functions as a cofactor in multiple bacterial dehydrogenases, oxidases and decarboxylases. This quinone has shown antioxidant properties and its presence has been shown in plant and animal products. Enzymes containing $P Q Q$ as a cofactor have been called quinoproteins. $P Q Q$ promotes growth in plants and animals. Little is known about the presence of $P Q Q$ in higher organisms.

Keywords: $P Q Q$, extract, bacterial, eukaryotic, tissue, cycling-redox.

\section{INTRODUCTION}

The pyrroloquinoline quinone (PQQ) is a novel cofactor of certain bacterial enzymes. It was described for the first time in Methylobacterium extorquens associated to ethanol dehydrogenase (FloresEncarnación et al., 2004; Misra et al., 2012; Shen et al., 2012). So, PQQ has been reported to be associated with dehydrogenases and oxidases, functioning as an enzymatic cofactor in some Gramnegative bacteria (Flores-Encarnación et al., 2014). For example, PQQ-glucose dehydrogenase was isolated from Gluconobacter suboxydans and Klebsiella aerogenes. This enzyme was found to be associated to the cytoplasmic membrane and functionally linked to respiratory chain (Meyer et al., 2013). In more recent years, in animal cells it has been reported that PQQ has a protector effect in cardiac lesions; it also prevents liver injury eliminating free radicals due to its antioxidant potent properties (50-100 times more effective than ascorbic acid) (Akagawa et al., 2016; Feng et al., 2014; Jia et al., 2015; Rucker et al., 2009; Tao et al., 2007). PQQ is a polyphenolic compound found in plants, various foods and biological fluids (Flores-Encarnación et al., 2017; Noji et al., 2007). The present study aimed to seek evidence the presence of PQQ in eukaryotic tissue extracts.

\section{MATERIAL AND METHODS}

\subsection{Biological Material}

For the present study, 3 female Wistar rats weighing 150 to 200 grams were used. The organs of the rats (spleen, muscle tissue, brain, stomach, lung, heart, liver, kidney) were extracted by dissection in the laboratory under optimal conditions and bioethical guidelines. The biological samples were kept at $-30^{\circ} \mathrm{C}$ in sterile flasks for their conservation.

\subsection{Tissue Extracts}

Tissue extracts were obtained using 1 gram of each tissue. For this, the tissue was cut into 2 to $3 \mathrm{~mm}$ fragments and then mechanically homogenized in $10 \mathrm{~mL}$ of lysis solution. The lysis solution contained: $50 \mathrm{mM}$ Tris hydrochloride, $150 \mathrm{mM} \mathrm{NaCl}, 10 \%$ SDS, $0.3 \mathrm{mM}$ PMSF and pH 7.0. For homogenization, 10 to 12 pulses were applied for $30 \mathrm{~s}$, with intervals of $1 \mathrm{~min}$ in cold. Then, the homogenate was centrifuged at 3,500 r.p.m. in cold for $10 \mathrm{~min}$. The supernatant was recovered and 
the pellet was discarded. The supernatant was centrifuged at 8,000 r.p.m. for 5 min and then it was frozen at $-30{ }^{\circ} \mathrm{C}$ until use.

\subsection{PQQ Quantification}

The quantification of PQQ was carried out according to the methodology reported by Paz et al. (1991). For this, glycine-borate solution, nitro blue tetrazolium (NBT) and the 'cycling redox' technique were used. The reaction mixture contained glycine-borate solution ( $\mathrm{pH} \mathrm{10),} \mathrm{Triton} \mathrm{X-100,}$ NBT and $100 \mu$ lof tissue extract. The reaction mixture was incubated at room temperature for 1 hour (in the dark) and the absorbance at $605 \mathrm{~nm}$ was measured. PQQ from Sigma-Aldrich Co. as standard was used.The analyses were conducted in triplicate.

\section{RESUlts}

As mentioned earlier, the spleen, muscle tissue, brain, stomach, lung, heart, liver, kidney were extracted from 3 female Wistar rats weighing 150 to 200 grams. Tissue extracts were obtained by mechanical homogenization and the debris was removed by centrifugation at 3,500 r.p.m. for 10 min. The supernatant obtained was centrifuged again at 8,000 r.p.m. for 5 min.This is how tissue extracts were obtained. The quantification of PQQ was carried out using the 'cycling redox' technique, which is based on the formation of a colorful complex of formazan using nitro blue tetrazolium. So, PQQ at alkaline $\mathrm{pH}$ can oxidize glycine in an amino oxidation reaction. The hydroquinones formed react with $\mathrm{O}_{2}$ to form superoxide radical which is followed by the reduction of nitro blue tetrazolium to formazan. The results are shown in Table 1. As shown in Table 1, using the cycling-redox technique in all the extracts tested, the presence of PQQ was detected. In this study, the liver, spleen and lung contained about $180 \mathrm{nmol}$ PQQ gram $^{-1}$ of tissue, while muscle tissue, brain and heart contained about 220-240 nmol PQQ gram $^{-1}$ of tissue. The stomach and liver registered the highest values in the content of PQQ, thus the values found were of 260-280 nmol PQQ gram $^{-1}$ of tissue. In addition to the above, other tests were carried out to verify the presence of PQQ. Thus, through a spectrophotometric scan between 300 and $400 \mathrm{~nm}$, signals were observed at 324, 329 and $353 \mathrm{~nm}$ which suggested the presence of PQQ (data not shown).

\section{DISCUSSION}

Enzymes containing PQQ as a cofactor have been called quinoproteins (Rucker et al., 2009). Quinoproteins were initially described in bacteria functioning as dehydrogenases, oxidases and decarboxylases. These PQQ-dependent bacterial enzymes directly oxidize sugars, alcohols, and aldehydes, a process that takes place in the bacterial cytoplasmic membrane (Flores-Encarnación et al., 2004; Rucker et al., 2009). Some authors have reported that both vegetable and some animal products contain PQQ (Stites et al., 2000). Thus, it has been described that parsley, green tea, green pepper, papaya and other vegetables contain significant concentrations of PQQ, as well as natto made from fermented soybeans (Kumazawa et al., 1995). The present study shows some data related with the presence of PQQ in extracts of eukaryotic tissue. As mentioned earlier, the tissue extracts were obtained by mechanical homogenization from spleen, muscle tissue, brain, stomach, lung, heart, liver, kidney of Wistar rats. It was shown in Table 1, PQQ was detected in tissue rat extrats using the 'cycling redox' technique. So, liver, spleen and lung extracts contained about $180 \mathrm{nmol}$ PQQ gram $^{-1}$ of tissue; muscle tissue, brain and heart extracts contained about 220-240 nmol PQQ gram ${ }^{-1}$ of tissue; stomach and liver extracts contained about 260-280 nmol PQQ gram $^{-1}$ of tissue. The presence of PQQ was observed also spectrophotometrically registering signals at 324,329 and $353 \mathrm{~nm}$ (data not shown). In addition to vegetables, the presence of PQQ has been reported in high concentrations in products of animal origin, such as eggs and milk (Paz et al., 1991). This suggests that PQQ could be present in eukaryotic cells. This could explain the presence of PQQ in the animal tissue extracts tested in this study, that is, PQQ must come from plant foods and animal products. Another important source of PQQ could be the intestinal bacterial microflora. As reported, various enterobacteria are known to synthesize PQQ. Many bacteria produce it in large quantities and excrete it into the environment. The amount of PQQ excreted can vary between $1 \mu \mathrm{g} \mathrm{mL}^{-1}$ to $\mathrm{mg} \mathrm{mL}^{-1}$, which depends on the composition of the growth medium (McIntire et al., 1994). Apparently, PQQ is a novel quinone that promotes many benefits in the animal organisms in which this substance has been tested. Its neuroprotective role has been reported due it works as a powerful antioxidant (Flores-Encarnación et al., 2014; Huang et al., 2015; Murray, 2018; Ohwada et al., 2008; Rucker et al., 2009). PQQ promotes growth in plants and animals. PQQ is a novel vitamin-like compound that acts as an essential active factor in the 
functioning of mitochondria. In addition to its role in mitochondria, PQQ stimulates growth and serves as a cofactor for a special class of enzymes involved in cellular functions including cellular growth, development, differentiation and survival (Flores-Encarnación et al., 2014; Harris et al., 2013; Murray, 2018). It has been reported that the immune system seems particularly sensitive to low PQQ levels: PQQ deprivation is accompanied by multiple defects in immune function and loss of the ability of white blood cells to respond properly (Ikemoto et al., 2017; Murray, 2018; Rucker et al., 2009). PQQ is an effective antioxidant, protecting mitochondria against oxidative stress-induced lipid peroxidation, protein carbonyl formation and inactivation of the mitochondrial respiratory chain (Hwang and Willoughby, 2018). Although PQQ is not biosynthesized in mammals, trace amounts of PQQ have been found in human and rat tissues at picomolar to nanomolar levels, and an especially large amount has been found in human milk. Although the higher organisms do not seem to biosynthesize PQQ, this quinone appears to have a universal function among living organisms. In the present study, it was observed that without exogenous addition of PQQ, this quinone is present in different tissues, so PQQ must perform some function that should be explored later.

Table1. Presence of $P Q Q$ in extracts of eukaryotic tissue.

\begin{tabular}{|l|l|}
\hline Tissue extract & PQQ $\left(\mathbf{n m o l ~}_{\text { gram }}^{-1}\right)$ \\
\hline Kidney & $\mathbf{1 8 0}$ \\
\hline Spleen & $\mathbf{1 8 0}$ \\
\hline Lung & $\mathbf{1 8 0}$ \\
\hline Muscle tissue & $\mathbf{2 2 0}$ \\
\hline Brain & $\mathbf{2 4 0}$ \\
\hline Heart & $\mathbf{2 4 0}$ \\
\hline Stomach & $\mathbf{2 6 0}$ \\
\hline Liver & $\mathbf{2 8 0}$ \\
\hline
\end{tabular}

\section{CONCLUSION}

This study showed some evidence of the presence of PQQ in extracts obtained from different rat tissues. Apparently PQQ is a universal molecule that has been present in both bacterial cells and plant and animal cells. Its functions as a bacterial cofactor have been extensive, however its role as an antioxidant in animal cells has provided a neuroprotective role. Further studies are needed to determine the function of PQQ in each of the tissues tested.

\section{ACKNOWLEDGEMENTS}

We appreciate the enthusiastic collaboration and technical support of LBM. Yáñez-Falcón de la Barrera A.R. Thank to PRODEP and Facultad de Medicina-BUAP for the facilities provided for the development of this work.

\section{REFERENCES}

[1] Akagawa, M., Nakano, M. and Ikemoto, K. (2016). Recent progress in studies on the health benefits of pyrroloquinolinequinone. Biosc. Biotechnol. Biochem. 80:13-22.

[2] Feng, X., Haixia, Y., Jinyao, L. and Lu, C. (2014). Pyrroloquinoline quinone inhibits oxygen/glucose deprivation-induced apoptosis by activating the PI3K/AKT pathway in cardiomyocytes. Mol. Cel. Biochem. 386:107-115.

[3] Flores-Encarnación M., Cabrera-Aguilar I., Cabrera-Maldonado C., Nava-Nolazco R.M. (2017). The effect of pyrroloquinoline quinone (PQQ) on viability of human colorectal adenocarcinoma HT-29 cell line. Intern. J. Res. Studies in Biosci. 5:18-24.

[4] Flores-Encarnación M., González-Gutiérrez J.Y., Amador-Bravo D., Bravo-Juárez L.A. and CabreraMaldonado C. (2014). A bacterial novel quinone participating in multiple functions in higher organisms. Global Adv. Res. J. Medicine and Med. Sci. 3:132-138,

[5] Flores-Encarnación M., Sánchez-Cuevas M. and Ortiz-Gutiérrez F. (2004). Las PQQ-deshidrogenasas. Un novedoso ejemplo de quinoproteinas bacterianas. Rev. Lat. Microbiol. 46:47-59.

[6] Harris C.B., Chowanadisaia W., Mishchuka D.O., Satrea M.A., Slupskya C.M. and Rucker R.B. (2013). Dietary pyrroloquinoline quinone (PQQ) alters indicators of inflammation and mitochondrial-related metabolism in human subjects. J. Nutr. Biochem. 24:2076-2084.

[7] Huang Y., Chen N. and Miao D. (2015). Biological effects of pyrroloquinoline quinone on liver damage in Bmi-1 knockout mice. Experim. Therap. Med. 10: 451-458. 
[8] Hwang P. and Willoughby D.S. (2018). Mechanisms behind pyrroloquinoline quinone supplementation on skeletal muscle mitochondrial biogenesis: possible synergistic effects with exercise. J. Am. Coll. Nutr. 18:1-11.

[9] Ikemoto K., Mori S. and Mukai K. (2017). Synthesis and crystal structure of pyrroloquinoline quinol (PQQH2) and pyrroloquinoline quinone (PQQ). Acta Crystallogr. Sect B: Struct Sci. 73:489-497.

[10] Jia, D., Duan, F., Peng, P., Sun, L., Ruan, Y. and Gu, J. (2015). Pyrroloquinoline quinone suppresses liver fibrogenesis in mice. PLoS ONE. 10:e0121939

[11] Kumazawa T., Sato K., Seno H., Ishilt A. and Suzukit O. (1995). Levels of pyrroloquinoline quinone in various foods. J. Biochem. 307:331-333.

[12] McIntire W.S. (1994). Quinoproteins. The FASEB J. 8:513-519.

[13] Meyer M., Schweiger P. and Deppenmeier U. (2013). Effects of membrane-bound glucose dehydrogenase overproduction on the respiratory chain of Gluconobacter oxydans. Appl. Microbiol. Biotechnol. 97:34573466.

[14] Misra H.S., Rajpurohit Y.S. and Khairnar N.P. (2012). Pyrroloquinoline quinone and its versatile roles in biological processes. J. Biosci. 37:313-325.

[15] Murray M. (2018). Pyrroloquinoline quinone (PQQ): the next essential nutrient and supplement superstar. Nutrafoods. 17:125-129.

[16] Noji, N., Nakamura, T., Kitahata, N., Taguchi, K., Kudo, T., Yoshida, S., Tsujimoto, M., Sugiyama, T. and Asami, T. (2007). Simple and sensitive method for pyrroloquinoline quinone (PQQ) analysis in various foods using liquid chromatography/electrospray-ionization tandem mass spectrometry. J. Agric. Food Chem. 55:7258-7263.

[17] Ohwada K., Takeda H., Yamazaki M., Isogai H., Nakano M., Shimomura M., Fukui K. and Urano S. (2008). Pyrroloquinoline quinone (PQQ) prevents cognitive deficit caused by oxidative stress in rats. J. Clin. Biochem. Nutr. 42:29-34.

[18] Paz M.A., Flückiger R., Boak A., Kagan H.M, and Gallop M. (1991). Specific detection of quinoproteins by redox-cycling staining. J. Biol. Chem. 266:689-692.

[19] Rucker, R., Chowanadisai, W. and Nakano, M. (2009). Potential physiological importance of pyrroloquinoline quinone. Altern. Med. Review. 14:268-277.

[20] Shen Y.Q., Bonnot F., Imsand E.M., Rose Figura J.M., Sjölander K. and Klinman J.P. (2012). Distribution and properties of the genes encoding the biosynthesis of the bacterial cofactor, pyrroloquinoline quinone. Biochem. 51:2265-2275.

[21] Stites T.E., Mitchell A.E. and Rucker R.B. (2000). Physiological importance of quinoenzymes and the Oquinone family of cofactors. J. Nutr. 130:719-727.

[22] Tao, R., Karliner, J., Simonis, U., Zheng, J., Zhang, J., Honbo, N. and Alano, C. (2007). Pyrroloquinoline quinone preserves mitochondrial function and prevents oxidative injury in adult rat cardiac myocytes. Biochem. Biophys. Res. Comm. 363:257-262.

Citation: Flores-Encarnación, M, et.al., The Presence of Pyrrolo Quinoline Quinone (PQQ) in Eukaryotic Tissue Extracts. International Journal of Research Studies in Biosciences. 2021; 9(1): 16-19. DOI: https://doi.org/10.20431/2349-0365.0901002.

Copyright: () 2021 Authors. This is an open-access article distributed under the terms of the Creative Commons Attribution License, which permits unrestricted use, distribution, and reproduction in any medium, provided the original author and source are credited. 